\title{
Viscoelastic Suppression of Gravity-Driven Counterflow Instability
}

\author{
P. Beiersdorfer, D. Layne, and E. W. Magee \\ Lawrence Livermore National Laboratory, Livermore, California 94550, USA
}

\section{J. I. Katz ${ }^{*}$}

Department of Physics and McDonnell Center for the Space Sciences, Washington University, St. Louis, Missouri 63130, USA

(Received 26 August 2010; published 31 January 2011)

\begin{abstract}
Attempts to achieve "top kill" of flowing oil wells by pumping dense drilling "muds," i.e., slurries of dense minerals, from above will fail if the Kelvin-Helmholtz instability in the gravity-driven counterflow produces turbulence that breaks up the denser fluid into small droplets. Here we estimate the droplet size to be submillimeter for fast flows and suggest the addition of a shear-thickening or viscoelastic polymer to suppress turbulence. We find in laboratory experiments a variety of new physical effects for a viscoelastic shear-thickening liquid in a gravity-driven counterstreaming flow. There is a progression from droplet formation to complete turbulence suppression at the relevant high velocities. Thick descending columns show a viscoelastic analogue of the viscous buckling instability. Thinner streams form structures resembling globules on a looping filament.
\end{abstract}

DOI: 10.1103/PhysRevLett.106.058301

PACS numbers: $83.80 . \mathrm{Hj}, 83.60 . \mathrm{Fg}, 83.60 . \mathrm{Rs}$

Dense fluids, i.e., mineral suspensions called "mud" [1,2], are introduced into oil wells to provide hydrostatic pressure to offset hydrocarbon (oil and gas) fluid pressure in deep formations, stopping upward flow and reducing the fluid pressure at the surface to near ambient. If hydrocarbon is flowing upward in the well, there is a counterflow between the upwelling hydrocarbon and the descending mud. Successful top kill requires that the mud descend despite this counterflow. However, upwelling at speeds $\gtrsim 1 \mathrm{~m} / \mathrm{s}$, as in the uncontrolled Macondo well "blowout" in the Gulf of Mexico in 2010, may lead to a KelvinHelmholtz instability [3].

If instability is not suppressed, the dense fluid introduced at the top of the column will be dispersed into small droplets that are spat out with the upwelling flow. In a "blown-out" oil well the consequence would be the failure of the dense fluid to accumulate to a depth sufficient to provide the hydrostatic pressure head necessary to "kill" (suppress the entry of hydrocarbon into) the well.

The purpose of this Letter is to report experiments demonstrating that a surrogate mud consisting of a shearthickening viscoelastic suspension is not dispersed by oil-mud counterflow. There appear to be no previous studies of two-phase flows in which one of the fluids is viscoelastic. Conventional drilling muds are typically Bingham plastics that flow as shear-thinning (pseudoplastic) fluids above a small elastic yield stress $[1,2,4,5]$. Such fluids are likely to be dispersed into small pockets or droplets and carried out of the well bore by a rapid counterflow. We demonstrate in experiments with viscoelastic shear-thickening fluids complete suppression of the Kelvin-Helmholtz instability at flow velocities and Reynolds numbers approaching those of the blown-out Macondo well.
At lower flow rates, thin filaments of immiscible fluid break up into droplets (the Plateau-Rayleigh instability [6]) under the influence of interfacial tension. We also demonstrate suppression of this instability in thin jets of viscoelastic fluid as elasticity prevents the rupture of the jet. In its place, we find novel "globule and filament" phenomenology.

We estimate the size of the droplets produced by turbulence, if it occurs, by assuming a Kolmogorov turbulent cascade [7] driven by the Kelvin-Helmholtz instability at an outer scale $L$ and velocity $U . L$ is taken as the diameter of the descending mud column (a fraction, determined by the mud flow rate, of the well bore diameter). $U$ is the velocity difference between oil and mud and must exceed the upwelling velocity of the oil for the mud to descend at all.

Adopting $U=3.7 \mathrm{~m} / \mathrm{s}$ [corresponding to Macondo's estimated [8] uncontrolled flow of 50000 barrels/day $(92 \mathrm{l} / \mathrm{s})$ through the $0.18 \mathrm{~m}$ diameter bore at the bottom of the well [9]] and $L=0.09 \mathrm{~m}$ (considering a mud column half the bore diameter) and taking a representative kinematic viscosity of crude oil of $10^{-5} \mathrm{~m}^{2} / \mathrm{s}$ [10] leads to a Reynolds number of roughly 40000 . Turbulence would be expected, but a well-developed inertial subrange [7] would not be. The problem is complicated by the presence of two dynamically interacting fluids with very different properties.

If the fluids are miscible, the inner Kolmogorov scale [7] provides an estimate of the scale of compositional heterogeneity:

$$
r_{\mathrm{visc}} \approx \nu^{3 / 4} \epsilon^{-1 / 4} \approx \nu^{3 / 4} L^{1 / 4} U^{-3 / 4},
$$

where $\epsilon \approx U^{3} / L$ is the specific energy dissipation rate and $\nu$ is the kinematic viscosity. The shear rate is high for 
$r>r_{\text {visc }}$ but decays $\propto \exp \left[-1.5\left(r / r_{\text {visc }}\right)^{-4 / 3}\right]$ for $r<r_{\text {visc }}$ [7]. Structure on the scale $r_{\text {visc }}$ develops in a time $\ll L / U$, but no finer spatial structure is expected. For miscible fluids the spatial structure does not take the form of spherical droplets because there is no interfacial energy acting to minimize the area of the boundary; we refer instead to "packets" of denser fluid.

The formation of a packet or droplet of one fluid surrounded by the other requires shear flow on the heterogeneity scale in both fluids. We adopt for the viscosity $\nu$ that of crude oil. For a comparatively inviscid, i.e., not shearthickening, mud, the characteristic size of the heterogeneity is $r_{\text {visc }} \sim 0.05 \mathrm{~mm}$. These denser mud volumes have a Stokesian descent (settling) velocity (for a density difference of $1 \mathrm{~g} / \mathrm{cm}^{3}$ ) $v_{\text {descent }}<1 \mathrm{~cm} / \mathrm{s}$, far less than the upward velocity of the fluid in which they are immersed. Even at the inner Kolmogorov scale the Reynolds stress $\rho u_{k}^{2} \sim \rho(\epsilon / k)^{2 / 3} \sim \rho(\epsilon \nu)^{1 / 2}$ far exceeds the Bingham yield stress $\lesssim 10 \mathrm{~Pa}$ of typical drilling muds [1] so that these muds behave essentially as shear-thinning (pseudoplastic) fluids.

If the fluids are immiscible, as is the case with waterbased muds, then interfacial energy further limits the droplet size, although it does not change the Kolmogorov inner scale of the turbulence within the homogeneous fluid. Equating the turbulent kinetic energy $\rho u_{k}^{2} / 2$ on a wave number scale $k$ in the volume of a droplet of radius $r_{\text {surf }}$ to its interfacial energy yields

$$
\frac{4 \pi r_{\text {surf }}^{3}}{3} \frac{\rho \epsilon^{2 / 3}}{2 k^{2 / 3}} \approx 4 \pi \sigma r_{\text {surf }}^{2} ;
$$

this is equivalent to the condition that the Weber number $\mathrm{We} \equiv \rho u_{k}^{2} r_{\text {surf }} / \sigma=\mathcal{O}(1)$, where $u_{k} \approx(\epsilon / k)^{1 / 3}$. By taking $k=1 / r_{\text {surf }}$,

$$
r_{\text {surf }} \approx\left(\frac{6 \sigma}{\rho}\right)^{3 / 5} \frac{L^{2 / 5}}{U^{6 / 5}} .
$$

The oil-water interfacial energy $\sigma \approx 0.025 \mathrm{~N} / \mathrm{m}$. The characteristic size of the droplets produced $r_{\text {surf }} \sim 0.7 \mathrm{~mm}$. The actual droplet sizes will be the greater of $r_{\text {visc }}$ and $r_{\text {surf }}$; $r_{\text {surf }}>r_{\text {visc }}$ for the assumed parameters. The turbulent Reynolds stress at the smallest turbulent scale exceeds the typical Bingham yield stress (by an even larger factor than for miscible fluids if evaluated at the scale $k=$ $\left.1 / r_{\text {surf }}\right)$. The Stokesian descent velocity $v_{\text {descent }} \sim$ $10 \mathrm{~cm} / \mathrm{s}$, again less than the upward velocity of the fluid in which the droplets are immersed (finite Reynolds number effects reduce the settling speed by a factor $\gtrsim 1$ ). The Weber number obtained from the settling speed $\mathrm{We}_{\text {descent }} \equiv \rho v_{\text {descent }}^{2} r_{\text {surf }} / \sigma \sim 0.3$, justifying the assumption that the droplets remain nearly spherical and intact.

For parameters over a broad range approximating the best estimates of those of the Macondo blowout, dense fluid introduced at the top of the well would not have sunk to the bottom but would have been swept out with the escaping crude oil. This explains the failure of top kill in that well. Similarly, had bottom kill, i.e., the introduction of dense fluid at the bottom of the well, been attempted while the well was flowing at $\approx 3 \mathrm{~m} / \mathrm{s}$, it would also have failed.

It is possible to suppress instability and thereby to avoid dispersion of the mud into small droplets and its sweepup by the counterflowing oil by adding to it a dilatant polymer with shear-thickening and viscoelastic properties. Before instability could grow to an amplitude sufficient to disperse the mud, the mud would have become very viscous or even elastic in the counterflow shear layer where dispersion would otherwise occur.

Corn starch-water emulsions are the classic shearthickening viscoelastic fluid $[11,12]$. Viscoelastic behavior is observed for mass ratios $\rho^{*}$ of corn starch to water in the range 1.1-1.7 (within this range there is little dependence on $\rho^{*}$ ). At low shear rates they shear thin from a viscosity of about $100 \mathrm{~Pa}$ s at $0.02 / \mathrm{s}$ to about $10 \mathrm{~Pa}$ s at $2-5 / \mathrm{s}$. Above this shear rate they abruptly shear thicken in a jamming transition to a dynamic viscosity $\eta_{\text {jam }} \gtrsim 1000 \mathrm{Pas}$. At these higher shear rates the emulsion also has an elastic response that is difficult to quantify, but that is manifested in the trampolinelike behavior of pools of corn starchwater emulsions.

The Kolmogorov inner scale turbulent shear rate $\sqrt{\epsilon / \nu} \approx U^{3 / 2} / \sqrt{L \nu} \sim 8000 / \mathrm{s}$ would far exceed that required for shear thickening and elasticity, so that the addition of corn starch to the mud would be expected to prevent its dispersal. Even the outer scale shear rate $U / L \sim 40 / \mathrm{s}$ at the mud-crude oil boundary is sufficient to put the mud in the shear-thickened viscoelastic regime and to suppress the Kelvin-Helmholtz instability.

A column of dense viscoelastic fluid is thus predicted to remain coherent, with its flow described by a (shearthickened) Reynolds number $\rho U L / \eta_{\text {jam }}=\mathcal{O}(1)$, sufficient to slow the Kelvin-Helmholtz instability and to preclude turbulence. Its descent would be retarded only by the viscous drag of the surrounding (Newtonian) lighter oil. As the column accelerates downward under the influence of its negative buoyancy, it would stretch into a thin filament, limited by its viscoelasticity.

In order to test these hypotheses we filled a transparent column $1.6 \mathrm{~m}$ tall and $63 \mathrm{~mm}$ in internal diameter with a transparent light mineral oil [13] of density $790 \mathrm{~kg} / \mathrm{m}^{3}$ and viscosity $6.4 \mathrm{mPas}$. Although the aspect ratio (depth to diameter) of the column was much less than that of a real oil well (several kilometers deep), the instability that would disperse the mud would be expected to occur in a comparatively short $[\mathcal{O}(10)$ diameters] length of the column, were it to occur at all. The subsequent flow would be expected to be gravity-driven settling, slow if the mud were dispersed but rapid if a coherent slug. Hence these experiments are applicable to any column or well with aspect ratio $\gg 1$.

We released 0.151 of water (with a dye added for visibility) from a funnel at a mean flow rate of $0.11 \mathrm{l} / \mathrm{s}$ and 
observed vigorous turbulence and dispersal of the stream into small (radius $\sim 1 \mathrm{~mm}$ ) droplets. The corresponding Reynolds number Re, based on the diameter of the water stream and the viscosity of the oil, was about 2000 at the speed $(1.15 \mathrm{~m} / \mathrm{s})$ of entry of the water into the oil, so turbulence was expected [14] (Re based on the viscosity of water was $\sim 10^{4}$ ). Aside from the volumetric flow rate and column diameter, the parameters, including the Atwood number At $\equiv\left(\rho_{\text {water }}-\rho_{\text {oil }}\right) /\left(\rho_{\text {water }}+\rho_{\text {oil }}\right)$, were comparable to those of drilling mud in a light crude oil; the predicted droplet radius $r_{\text {surf }} \approx 0.7 \mathrm{~mm}$. Unlike drilling muds, our fluids contained no surfactant, so most of these small globules rapidly coalesced once the turbulence decayed and descended in our static oil column [Fig. 1(a)].

We then repeated the experiment by using a strongly viscoelastic aqueous suspension $\left(\rho_{\text {susp }}=1.30 \mathrm{~g} / \mathrm{cm}^{3}\right.$ and At $=0.24$; these values are not far from those of drilling mud for which At $=0.33$ is representative) of $\rho^{*}=1.3$ mass ratio of corn starch to (colored) water extruded from a tube with inner diameter $12 \mathrm{~mm}$. A plunger was used to achieve a mass flow rate and velocity of the suspension close to the gravity-driven flow rate of water in the earlier experiment. The Kelvin-Helmholtz instability was suppressed [Fig. 1(b)], and the denser liquid descended as a coherent slug.

An instability analogous to the viscous buckling instability [15-18] began at the leading edge of the flow and led to buckling and clumping into a slug of low aspect ratio. The initial stage of this instability is visible in Fig. 1(b)

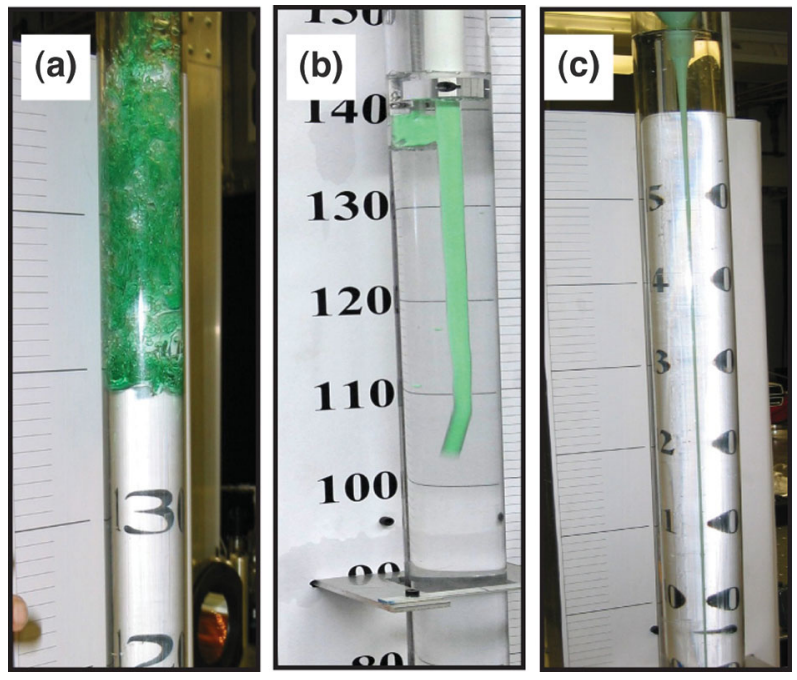

FIG. 1 (color). The left shows turbulent breakup following Kelvin-Helmholtz instability of descending water in oil column at $1.15 \mathrm{~m} / \mathrm{s}$ and $0.11 \mathrm{l} / \mathrm{s}$; the center shows descent of a coherent slug of viscoelastic suspension of corn starch in water (mass ratio $\left.\rho^{*}=1.3: 1\right)$ driven by a plunger at $0.56 \mathrm{~m} / \mathrm{s}$ and $0.11 \mathrm{l} / \mathrm{s}$ with stabilization of the instability; the right shows stable descent of the same suspension at 1-2 ml/s following passage of the leading edge. Vertical divisions are centimeters and tens of centimeters. between the 100 and $110 \mathrm{~cm}$ marks. The viscous buckling instability occurs at low Re. In our experiments Re is large in the oil (but not well-defined in the viscoelastic corn starch suspension). Instability is initiated by inertial forces at the leading edge of the descending slug rather than by viscous forces, and its growth is limited by the viscoelastic properties of the slug. Fragmentation of the descending column was observed only at flow speeds of $2.5 \mathrm{~m} / \mathrm{s}$ and produced slugs of width comparable to the initial column radius.

The descent speed in high Re flow of a spherical slug whose size is that of an oil well bore would be $\sim 2 \mathrm{~m} / \mathrm{s}$. A slug elongated vertically, as in Fig. 1(b), would descend faster. The Reynolds number in the oil is large enough that the drag force is described by a coefficient of turbulent skin friction $C_{\text {skin }} \approx 0.01$ [19], leading to a terminal descent velocity $v_{\text {descent }} \approx \sqrt{\Delta \rho g r /\left(C_{\text {skin }} \rho\right)} \approx 7 \mathrm{~m} / \mathrm{s}$ for representative $\Delta \rho / \rho=1$ and $r=50 \mathrm{~mm}$. This is fast enough to overcome the upwelling even in an unusually rapidly flowing well like Macondo. In our experiments thick and vertically elongated columns of dense viscoelastic fluid were affected by the inertial forces at their leading surfaces; much longer columns and greater fluid masses would be required to study this regime past the influence of the leading surface.

At much lower flow rates the denser aqueous viscoelastic fluid stretched into a thin straight vertical filament, cohering viscoelastically and thinning as it was accelerated by gravity. It did not disperse into drops of smaller diameter. In some trials, as illustrated in Fig. 1(c), it remained straight and unbroken for $\gtrsim 1 \mathrm{~s}$, demonstrating suppression of the Plateau-Rayleigh instability whose characteristic growth time $\approx 3 \sqrt{\rho r_{\mathrm{col}}^{3} / \sigma} \approx 7-20 \mathrm{~ms}$ for the column radius

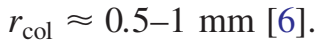

In order to study the transition between droplet formation (at $\left.\rho^{*}=0\right)$ and continuous vertical flow $\left(\rho^{*} \geq 1.2\right)$, we varied the suspension mass ratios and flow rates. A number of complex phenomena were observed. For example, at $\rho^{*}=1.2$ and flow rates of $1-2 \mathrm{ml} / \mathrm{s}$ the leading portion of the filament, after a steady descent of more than $1 \mathrm{~m}$, broke up into a complex flow of globules $(\approx 0.5 \mathrm{~cm}$ in diameter) strung together by thin filamentary loops, as shown in Fig. 2. This appears to be the result of the combined effects of the unstable dependence of flow rate on filament diameter (because of viscous drag by the surrounding fluid, in analogy to the instability of resistive hydraulic flow in open channels [20]) at low Reynolds numbers, the viscous buckling instability [15], the Plateau-Rayleigh instability [6], and viscoelasticity that inhibits breaking of the filaments even when they are greatly stretched and thinned.

We conclude that the addition of dilatant polymers to make a viscoelastic suspension is effective in suppressing both counterflow and surface tension instabilities and may, by making a Galilean transformation of reference frames, maintain the coherence of a body of descending 


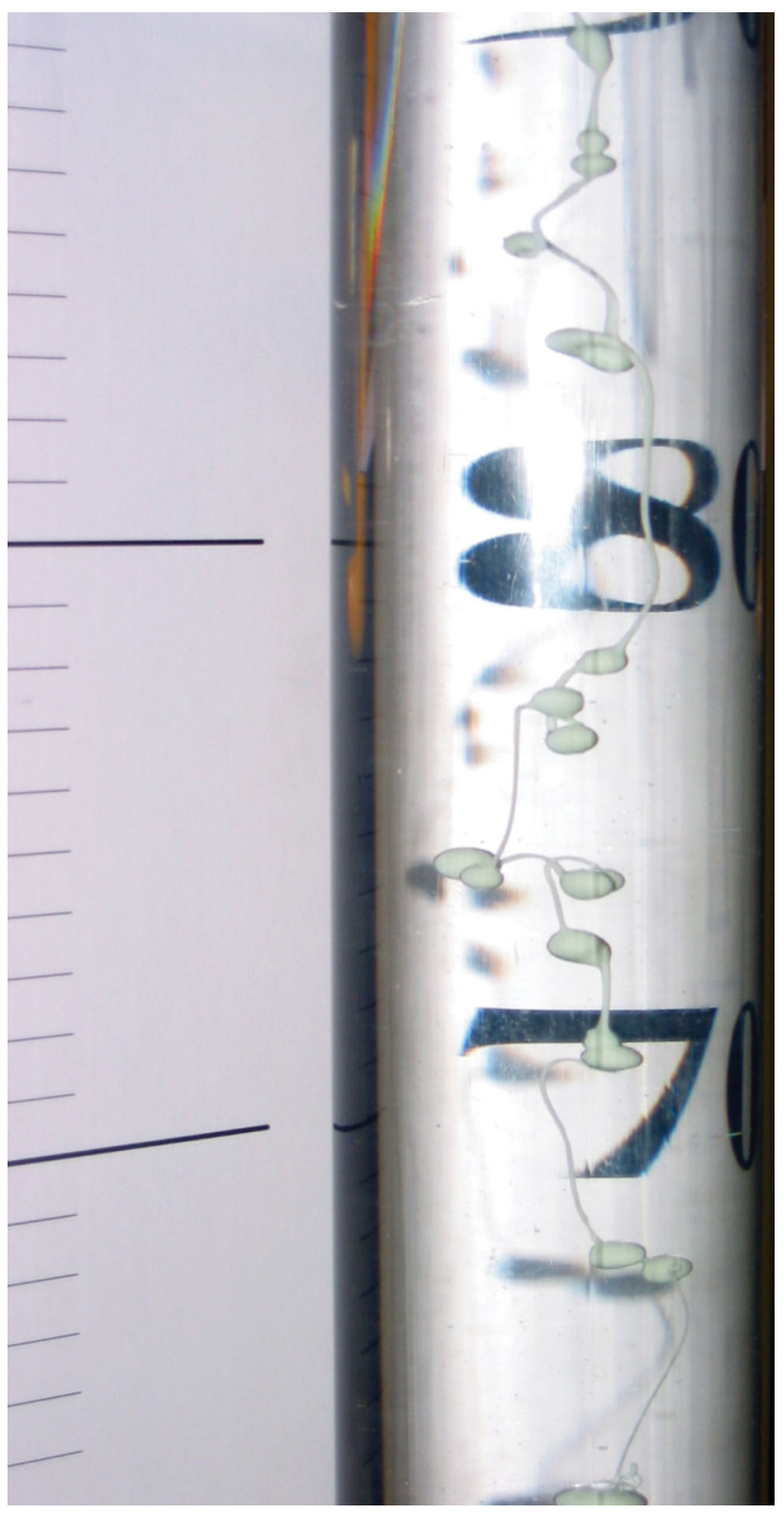

FIG. 2 (color). Descent of $\rho^{*}=1.2$ suspension at $1-2 \mathrm{ml} / \mathrm{s}$ showing globules bound by loops of viscoelastic filament. Vertical divisions are centimeters and tens of centimeters.

denser fluid in an upwelling flow. The potential practical application of these phenomena is the use of viscoelastic dense mud to fill a flowing (blown-out) oil well by enabling dense slugs of mud to descend against the upwelling oil. The hydrostatic pressure at the bottom may then be increased until it prevents entry of additional oil from the reservoir. This terminates the upwelling and kills the well. The phenomena observed go beyond this obvious practical application and focus attention on the rich physics of two counterstreaming fluids, one of which is shear-thickening and viscoelastic, which has so far received little attention.
We thank P. Dimotakis and R. Garwin for discussions that were the origin of this experiment and R. Grober for collaboration in a preliminary experiment with miscible fluids. This work was performed under the auspices of the U.S. DOE by LLNL under Contract No. DE-AC5207NA27344 and Laboratory Directed Research and Development Project No. 10-FS-005.

*Also at Lawrence Livermore National Laboratory, Livermore, CA 94550, USA.

katz@wuphys.wustl.edu

[1] H.C.H. Darley and G.R. Gray, Composition and Properties of Drilling and Completion Fluids (Butterworth-Heinemann, Woburn, MA, 1988), 5th ed.

[2] K. Van Dyke, Drilling Fluids (Petroleum Extension Service, University of Texas, Austin, 2000).

[3] G. K. Batchelor, An Introduction to Fluid Dynamics (Cambridge University Press, Cambridge, England, 1967).

[4] J.F. Steffe, Rheological Methods in Food Process Engineering (Freeman, East Lansing, MI, 1996), 2nd ed.

[5] W. C. Lyons and G.J. Plisga, Standard Handbook of Petroleum and Natural Gas Engineering (Elsevier, Amsterdam, 2005), 2nd ed.

[6] P. G. DeGennes, F. Brochard-Wyart, and D. Quéré, Capillary and Wetting Phenomena-Drops, Bubbles, Pearls, Waves (Springer, Berlin, 2002).

[7] H. Tennekes and J.L. Lumley, A First Course in Turbulence (MIT Press, Cambridge, MA, 1962).

[8] T. J. Crone and M. Tolstoy, Science 330, 634 (2010).

[9] U.S. Department of Energy Deepwater Horizon Response (http://www.energy.gov/open/oilspilldata.htm), accessed July 7, 2010.

[10] K. Van Dyke, Fundamentals of Petroleum (Petroleum Extension Service, University of Texas, Austin, 1997), 4th ed.

[11] F. S. Merkt, R. D. Deegan, D. I. Goldman, E. C. Rericha, and H. L. Swinney, Phys. Rev. Lett. 92, 184501 (2004).

[12] A. Fall, N. Huang, F. Bertrand, G. Ovarlez, and D. Bonn, Phys. Rev. Lett. 100, 018301 (2008).

[13] Multitherm 503 heat transfer fluid, MultiTherm Corp., Colwyn, PA 19023, USA (http://www.multitherm.com/ 503-phys-prop.html).

[14] R. Betchov and A. Szewczyk, Phys. Fluids 6, 1391 (1963).

[15] G. I. Taylor, in Proceedings of the Twelfth International Congress on Applied Mechanics (Springer, New York, 1968), pp. 382-388.

[16] L. Mahadevan and J. B. Keller, Proc. R. Soc. A 452, 1679 (1996).

[17] L. Mahadevan, W.-S. Ryu and D. T. Samuel, Nature (London) 392, 140 (1998).

[18] C. Dombrowski, B. Lewellyn, A. I. Pesci, J. M. Restrepo, J. O. Kessler, and R.E. Goldstein, Phys. Rev. Lett. 95, 184501 (2005).

[19] H. Schlichting and K. Gersten, Boundary-Layer Theory (Springer, Berlin 2000), 8th ed.

[20] R. F. Dressler and F. V. Pohle, Commun. Pure Appl. Math. VI, 93 (1953). 